\title{
Magnetic Resonance Imaging Features as Surrogate Markers of X-Linked Hypophosphatemic Rickets Activity
}

\author{
Marta Lempicki ${ }^{a} \quad$ Anya Rothenbuhler ${ }^{b}$ Valérie Merzoug ${ }^{a}$ \\ Stéphanie Franchi-Abella ${ }^{a} f \quad$ Catherine Chaussain ${ }^{c} d \quad$ Catherine Adamsbaum $^{a, e, f}$ \\ Agnès Linglart ${ }^{\mathrm{b}, \mathrm{e}, \mathrm{g}}$ \\ ${ }^{a}$ AP-HP, Bicêtre Paris-Sud Hospital, Department of Pediatric Radiology, Le Kremlin-Bicêtre, France; ${ }^{b}$ AP-HP, Bicêtre \\ Paris-Sud Hospital, Department of Pediatric Endocrinology, Diabetology and Reference Center for Rare Disorders \\ of Calcium and Phosphate Metabolism, Filière OSCAR and Platform of Expertise Paris-Sud for Rare Diseases, \\ Le Kremlin-Bicêtre, France; ${ }^{C}$ AP-HP, Bretonneau Hospital, Odontology Department, Paris, France; ${ }^{d}$ Paris Descartes

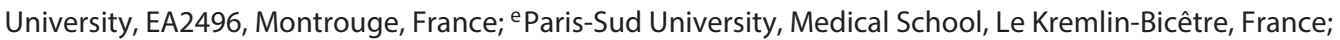 \\ ${ }^{\mathrm{f}}$ LTCI Telecom Paris Tech, Paris Saclay University, Le Kremlin-Bicêtre, France; 9 Inserm U1169, Bicêtre Paris-Sud \\ Hospital and Paris-Saclay University, Le Kremlin-Bicêtre, France
}

\section{Keywords}

X-linked hypophosphatemic rickets · Rickets .

Magnetic resonance imaging · Physis · Zone of

provisional calcification

\section{Abstract \\ Objective: X-linked hypophosphatemic rickets $(X L H)$ is the most common form of inheritable rickets. Rickets treatment is monitored by assessing alkaline phosphatase (ALP) levels, clinical features, and radiographs. Our objectives were to de- scribe the magnetic resonance imaging (MRI) features of $\mathrm{XLH}$ and to assess correlations with disease activity. Study Design: Twenty-seven XLH patients (median age 9.2 years) were included in this prospective single-center observation- al study. XLH activity was assessed using height, leg bowing, dental abscess history, and serum ALP levels. We looked for correlations between MRI features and markers of disease \\ C. Adamsbaum and A. Linglart contributed equally to this work.}

\section{KARGER}

(c) 2017 S. Karger AG, Basel

E-Mail karger@karger.com

www.karger.com/hrp activity. Results: On MRI, the median maximum width of the physis was $5.6 \mathrm{~mm}$ (range 4.8-7.8; normal <1.5), being $>1.5 \mathrm{~mm}$ in all of the patients. The appearance of the zone of provisional calcification was abnormal on $21 \mathrm{MRI}$ images (78\%), Harris lines were present on 24 (89\%), and bone marrow signal abnormalities were present on 16 (59\%). ALP levels correlated with the maximum physeal widening and with the transverse extent of the widening. Conclusions: MRI of the knee provides precise rickets patterns that are correlated with ALP, an established biochemical marker of the disease, avoiding $\mathrm{X}$-ray exposure and providing surrogate quantitative markers of disease activity.

(c) 2017 S. Karger AG, Basel

\section{Introduction}

$\mathrm{X}$-linked hypophosphatemic rickets (XLH) is the most common form of inheritable rickets [1]. First described by Albright et al. in 1937 [2], it affects approximately 1 in 
20,000 births [3]. XLH is transmitted by X-linked dominant inheritance and is mainly caused by mutations in the phosphate-regulating gene with homologies to endopeptidases on the $\mathrm{X}$ chromosome (PHEX) gene $[4,5]$. The lack of functional PHEX is associated with elevated circulating fibroblast growth factor 23 (FGF23) levels [6, 7]. FGF23, secreted by osteocytes, reduces serum phosphate by inhibiting proximal renal tubular phosphate reabsorption $[8,9]$, suppresses the expression of 1-alpha hydroxylase, and stimulates the expression of 24-hydroxylase, thereby limiting the circulating level of $1,25(\mathrm{OH})_{2} \mathrm{D}$. Elevated concentrations of circulating intact FGF23 favor renal phosphate wasting and hypophosphatemia and prevent the production of $1,25(\mathrm{OH})_{2} \mathrm{D}[10-12]$. Chronic hypophosphatemia leads to rickets by a combination of mechanisms, including a lack of endochondral ossification and impaired mineralization [13, 14]. Although in most cases the XLH phenotype in children is attributable to increased FGF23 activity, several features of the disease might also be the result of PHEX-deficient proteolytic processing of matrix proteins in mineralized tissues such as bone and teeth [15].

The symptoms of rickets include bowing deformity of the lower limbs, bone pain, short stature, and enlarged physes [16]. Spontaneous dental abscesses related to impaired dentin mineralization are frequently observed [17]. The biological abnormalities seen in untreated children with XLH include low serum phosphate and measurable urinary phosphate (commonly termed phosphate wasting), along with normal or low-normal serum calcium, moderately elevated parathormone, and inappropriately low levels of $1,25(\mathrm{OH})_{2} \mathrm{D}$. The alkaline phosphatase (ALP) level, a well-established biochemical marker of disease activity, is elevated in untreated patients $[16,18]$.

The current standard treatment is a combination of phosphate supplements several times daily and a vitamin $\mathrm{D}$ analog, i.e., 1-alpha hydroxylated vitamin D calcitriol or alfacalcidol $[16,18-20]$. The treatment of XLH in infants and children has a number of goals, including curing rickets, straightening the legs, and improving growth, though often these goals are not fully achieved. These challenges highlight the need for objective measures of disease activity in order to accurately guide the adjustment of the doses of vitamin D analogs and phosphate supplements. Physicians currently rely on a combination of clinical, radiological, and laboratory parameters, including growth velocity, orthopedic measures of leg bowing, semiquantitative evaluation of rickets on radiographs, and serum ALP levels; however, none of these markers alone - or in combination - has demonstrated accuracy or reproducibility.

Linear bone growth occurs secondary to endochondral ossification, which takes place in the growth plate, where chondrocytes are arranged in columns, surrounded by their matrix. As chondrocytes undergo differentiation and maturation, they maintain the same anatomical position, changing their morphology and the composition of the matrix they secrete as these processes occur. This explains the layered organization of the physis: the resting zone, the proliferative zone, and the hypertrophic zone. The hypertrophic chondrocytes ultimately undergo apoptosis, allowing the invasion of vascular elements of bone marrow as well as mineralization of the secreted extracellular matrix (ECM) [21]. The zone of provisional calcification (ZPC) is adjacent to the hypertrophic zone and consists of highly mineralized ECM.

While plain radiograph patterns in XLH have been well described in previous reports [22, 23], to our knowledge, there have been no cohort studies focusing on magnetic resonance imaging (MRI) patterns during the course of XLH. On the other hand, MRI is not only an excellent imaging tool but also a non-irradiating imaging technique, which is an important factor in a pediatric population [24]. The primary objective of our study was therefore to provide a detailed description of the MRI features of the distal femur in XLH. The secondary objective was to assess the correlation of MRI patterns with disease activity, based on clinical and laboratory markers, in order to determine whether MRI patterns could serve as a surrogate marker of disease activity.

\section{Patients and Methods}

\section{Patients}

This prospective descriptive single-center observational study was conducted at the French Reference Center for Rare Disorders of Calcium and Phosphate Metabolism (Bicêtre Paris-Sud Hospital) from October 2012 to December 2013. Twenty-seven children affected by XLH were included. To be eligible for inclusion, patients were required to (1) be under the age of 18 years, (2) be monitored at the French Reference Center for Rare Disorders of Calcium and Phosphate Metabolism, (3) present with rickets due to XLH and have a PHEX mutation, (4) currently be on phosphate supplements and alfacalcidol, (5) consent to an MRI scan of the distal left femur (or, if not possible, the distal right femur), and (6) not require sedation. The MRI protocol consisted of a 3-dimensional spectral attenuated inversion recovery (SPAIR) sequence $(\mathrm{TR}=1,400, \mathrm{TE}=50$, slice thickness $=1.12 \mathrm{~mm})$ with $3-\mathrm{mm}$ multiplanar reconstructions, and a frontal T1-weighted turbo spin echo sequence $(\mathrm{TR}=629, \mathrm{TE}=17$, slice thickness $=3.5 \mathrm{~mm})$, on a Philips Achieva 1.5-T MRI system. 
Table 1. Patient characteristics $(n=27)$

\begin{tabular}{|c|c|c|c|}
\hline & Distribution & $\begin{array}{l}\text { Median } \\
\text { (5th and 95th percentile) }\end{array}$ & Normal range \\
\hline \multicolumn{4}{|l|}{ Sex } \\
\hline Girls & $16(59 \%)$ & & \\
\hline Boys & $11(41 \%)$ & & \\
\hline Age at MRI, years & & $9.2(7.6 ; 10.9)$ & \\
\hline \multicolumn{4}{|l|}{ Height } \\
\hline Short stature & $7(26 \%)$ & $-0.89(-1.27 ;-0.50) \mathrm{SD}$ & $-2 \mathrm{SD}<$ height $<2 \mathrm{SD}$ \\
\hline Within normal range & $20(74 \%)$ & & \\
\hline \multicolumn{4}{|l|}{ Deformity } \\
\hline Varus & $13(48 \%)$ & ICD: $3.2(1.3 ; 5.0) \mathrm{cm}$ & \\
\hline Valgus & $7(26 \%)$ & IMD: $1.8(0.5 ; 3.0) \mathrm{cm}$ & \\
\hline Straight legs & $7(26 \%)$ & & \\
\hline \multicolumn{4}{|l|}{ Dental abscess history } \\
\hline Yes & $12(44 \%)$ & & \\
\hline No & $15(56 \%)$ & & \\
\hline \multicolumn{4}{|c|}{ Serum alkaline phosphatase level } \\
\hline Elevated & $13(48 \%)$ & $349(315 ; 443) \mathrm{IU} / \mathrm{L}$ & 200-350 IU/L \\
\hline Normal & $14(52 \%)$ & & \\
\hline
\end{tabular}

The local institutional review board approved the study. All patients and parents gave their informed consent before inclusion in the study.

Clinical and Laboratory Evaluation of XLH Activity (Table 1)

The following data were recorded for each patient: sex, age at MRI, height (converted to a standard deviation [SD] score), weight (for age and sex expressed as percentile of the normal reference population), absence or presence and type of leg bowing, intercondylar (genu varum) or intermalleolar (genu valgum) distances, presence or absence of a history of dental abscess, and serum ALP level at the time of MRI. The level of ALP activity was measured by colorimetric analysis.

\section{Imaging Evaluation}

Radiographs. Twenty-four patients had a frontal radiograph of the distal left femur within 1 year of the MRI in the course of their standard follow-up (mean: 3.6 months [0 day to 1 year]). We studied the physeal widening using the reference atlas if needed [25]. We also studied the appearance of the ZPC. In healthy children, the ZPC appears as a continuous transverse radiodense band $<1 \mathrm{~mm}$ thick $[26,27]$ (Fig. 1a, b). In XLH patients, we expected a lack of ZPC sharpness, i.e., an ill-defined or widened pattern of the ZPC (Fig. 1c). To quantify the severity of rickets on radiographs, we decided not to apply the Thacher score [28] because it was created to assess nutritional rickets and for instance includes the assessment of radiolucency, a feature rarely present in XLH.

MRI. Twenty-seven children with XLH underwent MRI of the knee (25 left knees and 2 right knees). The features studied were:
(1) The maximum width of the physis. This was measured on a sagittal SPAIR image, at the widest part of the physis, along the long axis of the femoral diaphysis (Fig. 2a). The medial or lateral location where the physeal widening was greatest was noted. (2) The transverse extent of the widening. This was measured on a frontal SPAIR image and expressed as the ratio between the transverse length of the widened portion of the physis and the total transverse length of the physis (Fig. 2b). (3) The continuity and regularity of the ZPC. This was considered normal when continuous and regular (Fig. 2c). It was considered discontinuous when not visible for at least $5 \mathrm{~mm}$ in any direction, and irregular when saw-toothed over more than $10 \mathrm{~mm}$ on 2 adjacent slides (Fig. 2a, b). (4) Any bone signal abnormality studied in a coronal plane and defined as a hyperintense signal on SPAIR and hypointense signal on T1-weighted sequences. To be considered significant, pathological signal abnormalities had to be clear, to distinguish them from the heterogeneous signal due to hematopoietic to fatty marrow conversion [29]. The side was recorded, as was the metaphyseal or epiphyseal predominance (Fig. 2d-g). (5) The presence or absence of Harris lines on the T1-weighted coronal plane image. Harris lines are metaphyseal lines which are parallel to the physis, and are hypointense on all sequences (Fig. 2h) [30]. (6) All other structural abnormalities, such as osteochondritis.

Both radiographic and MRI features were assessed by consensus (M.L., 5 years of experience, and C.A., 25 years of experience).

\section{Statistical Analysis}

Statistical analysis was performed using GraphPad Prism 5.0. Data are presented as medians (5th to 95 th percentile). The Mann- 

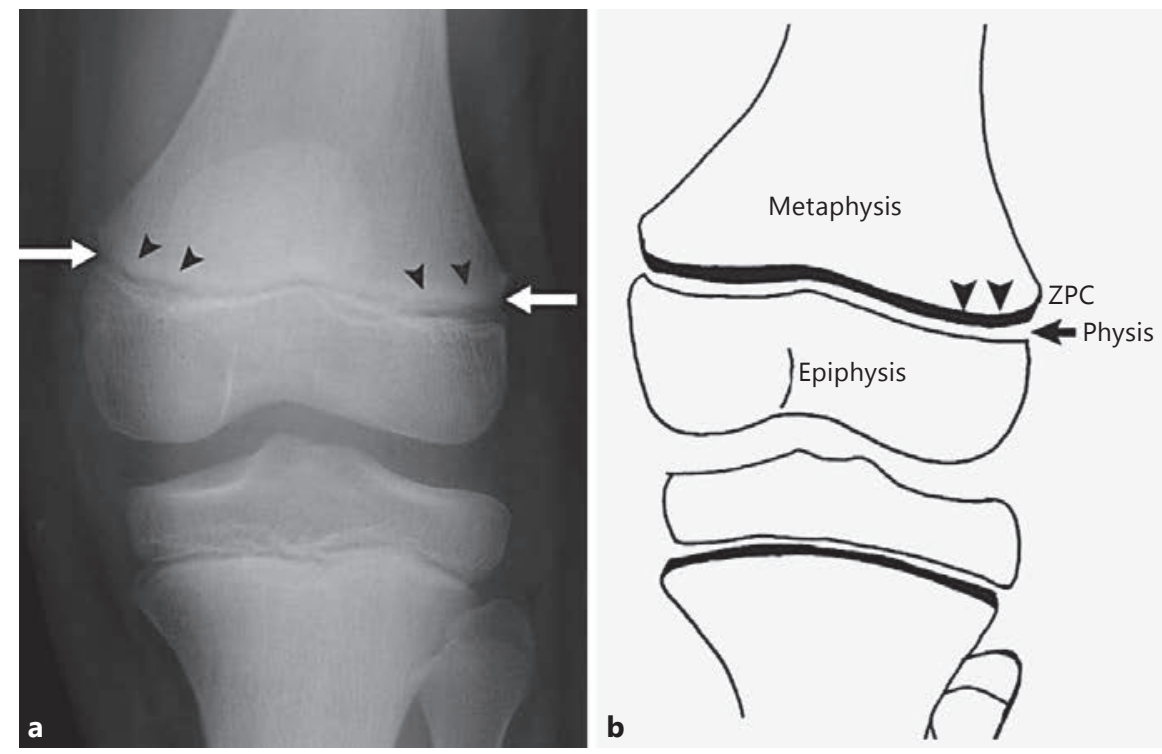

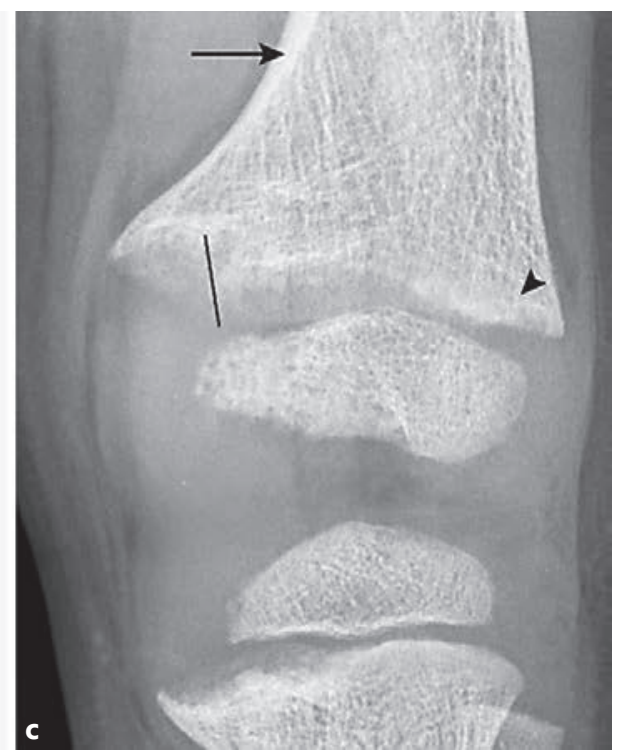

Fig. 1. X-linked hypophosphatemic rickets $(\mathrm{XLH})$ and normal radiographic patterns. a, b Normal pattern of the left knee on a frontal plain radiograph (a) and a drawing (b). Routine radiograph of a healthy 9 -year-old boy. The physis (arrows) appears as a radiolucent flat disc between the epiphysis and the metaphysis. The normal zone of provisional calcification (ZPC) (arrowheads) is a con- tinuous, regular, thin $(<1 \mathrm{~mm}$ thick) radiodense band. c Frontal radiograph of a boy aged 3 years and 5 months treated for XLH. The ZPC (arrowhead) is ill defined, and the physis (solid line) is widened. Note the preserved cortical bone (arrow) and the coarse trabecular bone.
Whitney test was used when comparing skewed variables between groups of patients. Correlations of clinical features with MRI patterns were performed using the Fisher exact test.

\section{Results}

\section{Patient Characteristics}

Table 1 summarizes the clinical and laboratory features of the 27 XLH patients included (16 girls and 11 boys, consistent with the expected sex ratio of $1.4 / 1$ for diseases with X-linked dominant inheritance). The median age was 9.2 years (range 7.6-10.9).

At the time of the study, 7 children (26\%) had short stature (i.e., height SD $<-2$ SD), and 20 (74\%) presented with leg bowing (either varus [13,48\%] or valgus [7,26\%] deformities); none of the participants had undergone leg surgery prior to the study. Twelve children (44\%) had experienced at least 1 dental abscess, and 18 (67\%) had ALP levels above the upper normal range for their age despite treatment. All the children were being treated with optimal doses of alfacalcidol and phosphate supplements at the time of the study.

\section{Radiographic Findings, Distal Femur $(n=24)$}

(Table 2)

Twenty-four of the 27 children (89\%) had an anteroposterior knee radiograph, which confirmed abnormal physes in all patients. Of these, 20 (83\%) showed widening of the physis, and 22 (92\%) showed an ill-defined ZPC (Fig. 3b). Two patients (8\%) had physeal widening while the ZPC appeared normal. Four patients $(17 \%)$ had a thin physis without any widening, but an unclear ZPC; all of those patients were $>13$ years of age. Finally, 18 patients $(75 \%)$ had both physeal widening and an ill-defined ZPC. In 23 patients (96\%), the abnormalities were predominantly medial, being similar on the medial and lateral sides in only in 1 case.

\section{MRI Findings $(n=27)$ (Table 2)}

The median maximum width of the femoral physis was $5.6 \mathrm{~mm}$ (range $4.8-7.8$ ). The maximum physeal width was $>1.5 \mathrm{~mm}$ - considered as the threshold for normal [31] - in all of the children (100\%). The maximum width was located on the medial side of the femur on 14 MRI images (52\%) and on the lateral side on $13(48 \%)$. The median transverse extent of the widening (as percentage of the total transverse length of the physis) was 55\% (range 42.9-66.2\%). 

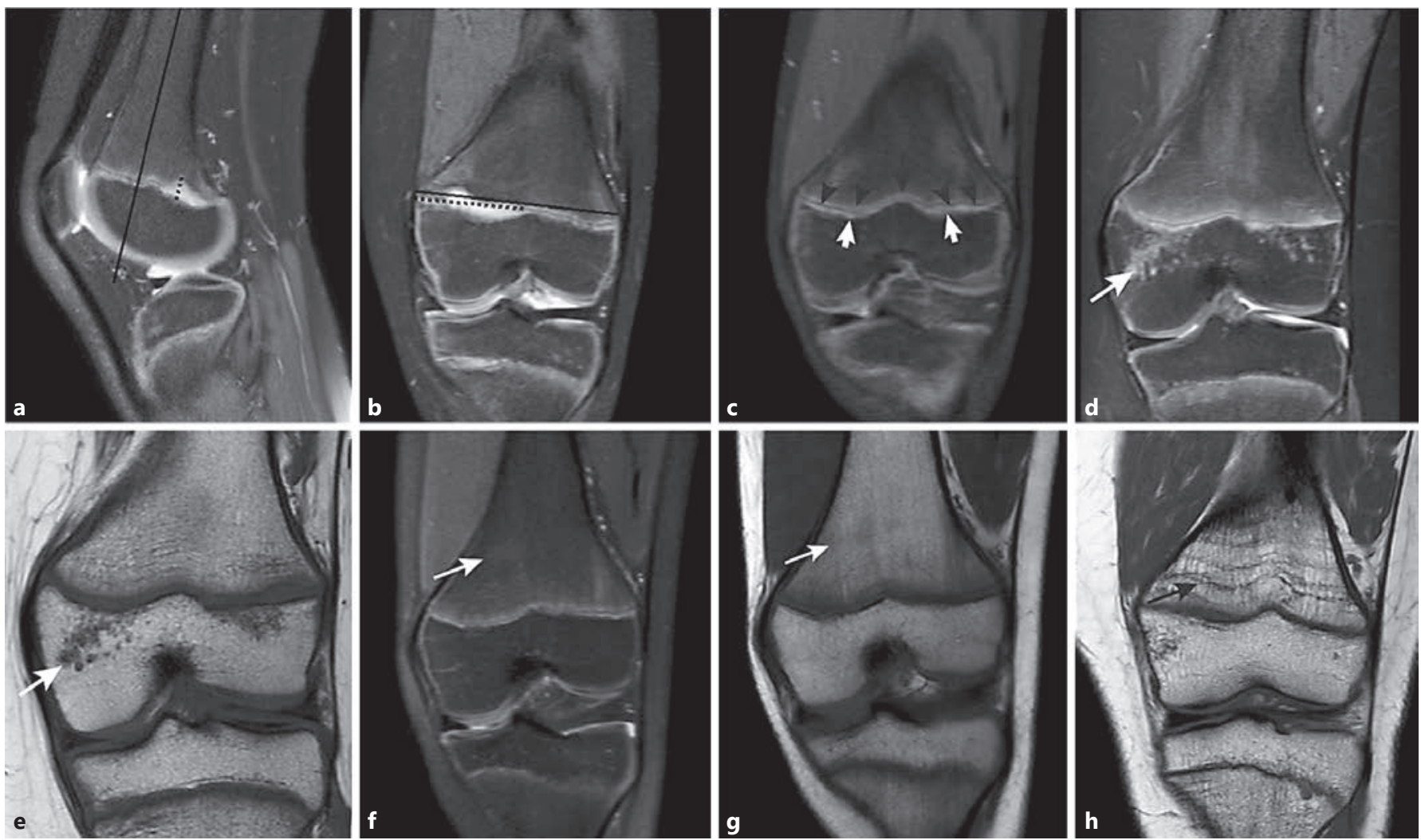

Fig. 2. Magnetic resonance imaging features of X-linked hypophosphatemic rickets patients. a Physeal width: measurement of the widest portion of the physis (dotted line) along the long axis of the femoral shaft (solid line) on a sagittal spectral attenuated inversion recovery (SPAIR) slice. The zone of provisional calcification (ZPC) is not well seen in this area (compare with c). b Transverse extent of the physeal widening: defined as the ratio between the transverse length of the widened portion of the physis (dotted line) and the total transverse length of the physis (solid line) on a frontal SPAIR slice. c Normal pattern of the ZPC (arrowheads). Frontal SPAIR slice. Regular, continuous, thin hypointense line be-

tween the hyperintense cartilaginous physis (white arrows) and the hyperintense newly formed bone of the metaphysis (black arrow). d, e Bone marrow abnormalities of the epiphysis: irregular areas of hyperintensity on SPAIR sequences (d) and hypointensity on T1-weighted sequences (e) (arrows). f, g Nonpathological heterogeneous signal due to hematopoietic to fatty marrow conversion, seen as mild hyperintensity on SPAIR sequence (f) and mild hypointensity on T1-weighted sequence (g) (arrows). h Harris lines (arrow): thin transverse metaphyseal lines, appearing hypointense on all sequences, shown on a frontal T1-weighted slice.

The ZPC was abnormal on 21 MRI images (78\%), being discontinuous in 14 (52\%) and irregular in 19 (70\%) cases (Fig. 3a, c). Harris lines were present in most cases (24/27 images, $89 \%$ ) (Fig. 3d). Bone marrow signal abnormalities were detected on $16 / 27$ of the MRIs (59\%) (Fig. 3a, c). They were predominantly epiphyseal on $14 / 16$ images (88\%) and were predominantly medial in all but 1 case.

None of the children had a physeal width $\leq 1.5 \mathrm{~mm}$. Two children (7\%) showed an osteochondritis pattern on the medial femoral condyle (Fig. 3e, f).

Correlations with Clinical and Laboratory Parameters

Higher levels of serum ALP were positively associated with the maximum physeal width $(p<0.05)$ and the transverse extent of the widened physis $(p<0.05)$ (Table 3$)$. On the other hand, we found no correlation between serum ALP levels and the ZPC pattern, the presence of Harris lines, or metaphyseal or epiphyseal signal abnormalities. Moreover, we failed to find any correlation between the other clinical parameters and either the maximum physeal widening or the transverse extent of the physeal widening.

There was no statistical correlation between the presence of a signal abnormality and that of a knee deformity 
Table 2. Radiographic and MRI findings

\begin{tabular}{|c|c|c|}
\hline & Radiographic $(n=24)$ & MRI $(n=27)$ \\
\hline Physeal widening & $20(83 \%)$ & $27(100 \%)$ \\
\hline Median maximum width, $\mathrm{mm}$ & & $5.6(4.8-7.8)$ \\
\hline \multicolumn{3}{|l|}{ Side of predominant abnormalities } \\
\hline Medial & $23(96 \%)$ & $14(52 \%)$ \\
\hline Both medial and lateral & $1(4 \%)$ & \\
\hline Lateral & & $13(48 \%)$ \\
\hline Median transverse extent of widening & & $54 \%(42.9-66.2 \%)$ \\
\hline Ill-defined ZPC & $22(92 \%)$ & $21(78 \%)$ \\
\hline Physeal widening and ill-defined ZPC & $18(75 \%)$ & \\
\hline Physeal widening and normal ZPC & $2(8 \%)$ & \\
\hline No physeal widening, ill-defined ZPC & $4(17 \%)$ & \\
\hline Harris lines & & $24(89 \%)$ \\
\hline Signal abnormalities reflecting bone marrow edema & & $16(59 \%)$ \\
\hline \multicolumn{3}{|l|}{ Side of predominant abnormalities } \\
\hline Medial & & $15(94 \%)$ \\
\hline Both medial and lateral & & $1(6 \%)$ \\
\hline
\end{tabular}

$(p=0.08$, odds ratio $=4.37[0.66-72.53])$, or between the type of deformity (valgus or varus) and the side of the signal abnormality, when there was one $(p=1)$.

\section{Discussion}

The cause and mechanisms of XLH were deciphered only very recently, with the discovery of the crucial role of FGF23 in phosphate handling, and hence tissue mineralization.

Today, the standard treatment aims to restore physiological $1,25(\mathrm{OH})_{2} \mathrm{D}$ levels and maintain serum phosphate levels through the use of vitamin D analogs and phosphate supplements. However, multiple factors acting together hinder complete healing of rickets in children with XLH. These include genetic heterogeneity of the disease, poor treatment adherence related to the requirement to take multiple daily medications and the unpleasant taste of phosphate supplements, the occurrence of hypercalciuria and hyperparathyroidism, and the absence of functional PHEX in the ECM. The cohort of patients described in the current report accurately reflects children with XLH managed in 2012-2013 in a reference center providing multidisciplinary care. Sex ratio, stature, and bone deformities are similar to those described in other reports [32-34].

Our study was designed to provide state-of-the-art MRI patterns of the bone and growth plate of XLH patients receiving the "standard treatment," and to identify novel surrogate markers of rickets activity in this population. As highlighted above, novel therapies such as antibodies neutralizing FGF23 are eagerly awaited, since they have shown promising results in adults with XLH [35, 36]. It is therefore of importance to accurately assess the natural history of the disease and to quantitatively measure the effect of such therapies on disease activity. Currently, MRI is the best imaging tool for this, as it is a nonirradiating technique providing exquisite anatomic details [37].

MRI, with its better resolution and 3-dimensional acquisition, is a much more precise tool than standard radiography for evaluating physeal widening and its transverse extent. On MRI, the normal physis appears trilinear on water-sensitive sequences. The distal femoral physis is hyperintense, the ZPC is a thin hypointense line, and the vascular primary spongiosa of the metaphysis is hyperintense on T2-weighed sequences [24]. 

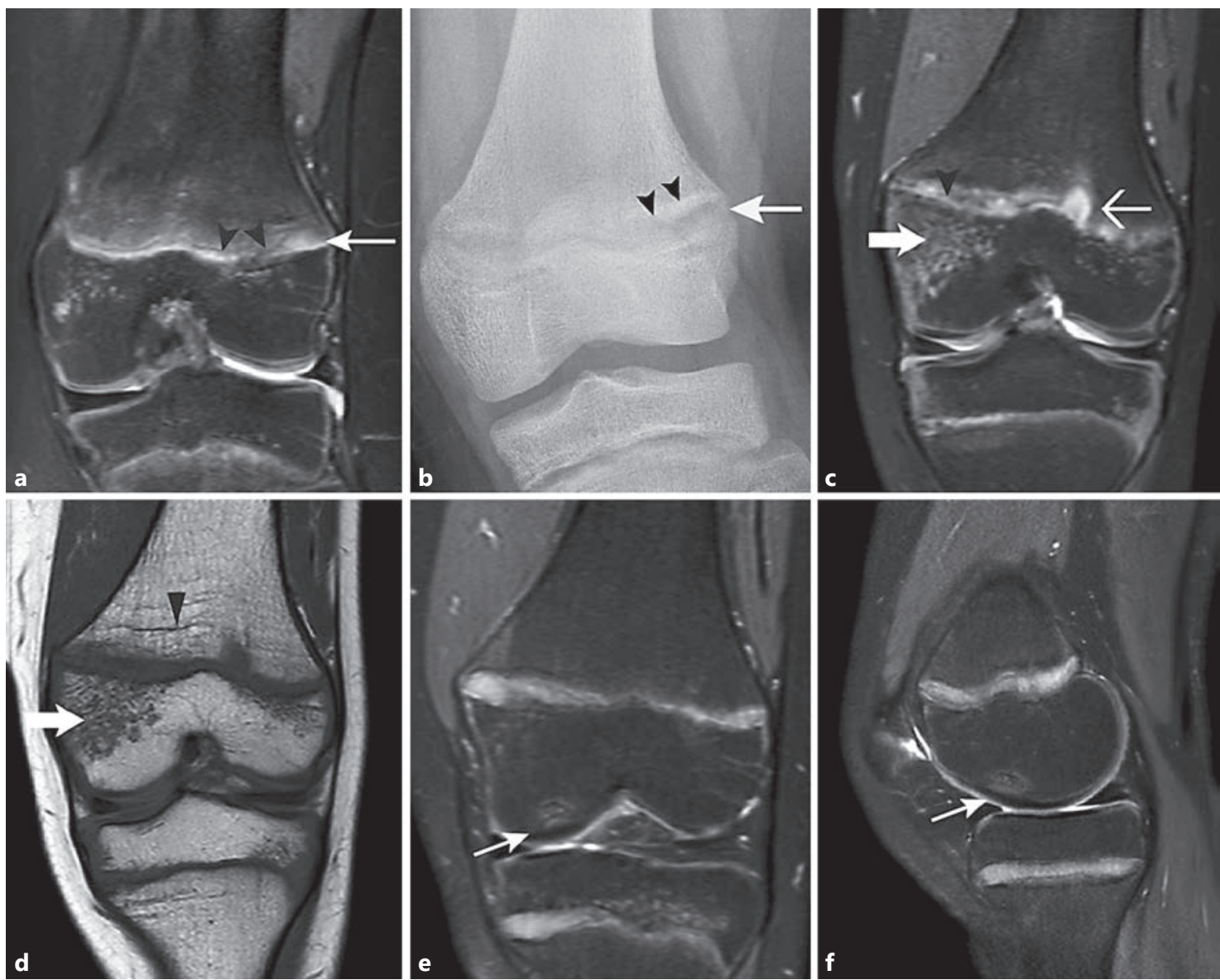

Fig. 3. Radiographic and magnetic resonance imaging (MRI) patterns of X-linked hypophosphatemic rickets (XLH) patients. a, b Frontal spectral attenuated inversion recovery (SPAIR) MRI image (a) and radiograph (b) of a 13-year-old boy treated for XLH. On the radiograph, both the zone of provisional calcification (ZPC) (arrowheads) and physis (arrows) appear ill defined, whereas MRI enables precise analysis of these regions. c, $\mathbf{d}$ MRI findings in an 11-year-old boy treated for XLH. Frontal SPAIR (c) and T1- weighted (d) sequences. The physis is enlarged (thin arrow), and the ZPC is discontinuous and irregular (arrowhead in c). Note the epiphyseal signal abnormalities (thick arrows) and the metaphyseal Harris lines (arrowhead in d). e, f Frontal SPAIR MRI image. Medial femoral osteochondritis (arrows) in an asymptomatic 13-year-old girl. Round-shaped abnormal hyperintensity of the subchondral area.

Table 3. Associations between disease activity and quantitative MRI findings

\begin{tabular}{|c|c|c|c|}
\hline Median [5th and 95th percentile] & $\begin{array}{l}\text { Patients with ALP } \\
<349 \text { IU/L }\end{array}$ & $\begin{array}{l}\text { Patients with ALP } \\
>349 \text { IU/L }\end{array}$ & $p$ value \\
\hline Number of patients & 13 & 14 & \\
\hline Age, years & $8.1[7.0 ; 11.1]$ & $10.6[6.5 ; 12.3]$ & 0.404 \\
\hline Height SD & $-1.0[-1.6 ;-0.5]$ & $-0.5[-1.3 ;-0.1]$ & 0.158 \\
\hline Maximum physeal widening, $\mathrm{mm}$ & $4.5[3.3 ; 6.2]$ & $7.2[5.3 ; 10.5]$ & 0.010 \\
\hline Percentage of total transverse physeal length showing widening & $39[30 ; 58]$ & $70[47 ; 85]$ & 0.026 \\
\hline
\end{tabular}

Disease activity was assessed by serum ALP at the time of the MRI. ALP, alkaline phosphatase; MRI, magnetic resonance imaging; $\mathrm{SD}$, standard deviation. 
To increase the sensitivity of the MRI features as a biomarker, we focused our study on the distal end of the femur, where rapid growth occurs. We were able to identify the location of the physeal widening as either medial or lateral on MRI, though it appeared to be medial on all but 1 of the radiographs. It should be noted, however, that when there was major physeal widening on MRI (>7 $\mathrm{mm}, 10$ patients), it was always predominantly medial. This may be explained by the impact of mechanical load and stress (i.e., the number of chondrocytes, their rate of proliferation, the extent of chondrocyte hypertrophy, and the synthesis and degradation of the ECM) on the physis due to the abnormal shape of the legs [38]. Impaired bone mineralization causes progressive leg bowing and anteromedial rotation of the tibiae [16], leading to a greater mechanical load on the medial compartment of the knee.

As expected in rickets, $100 \%$ of our patients were observed to have physeal widening on MRI, which suggests a lack of apoptosis of the hypertrophic chondrocytes and thus impaired endochondral ossification [39]. The fact that this process is common to all forms of rickets [13] explains why widening of the physis is the main radiological feature observed in rickets, whatever the etiology [31]. Interestingly, we found a significant correlation between the widening of the physis and its transverse extent and serum ALP, and hence disease activity.

This finding underlines the fact that even with straight legs and controlled ALP levels, patients may have signs of rickets on MRI. That is, despite good prescribing and adherence, the standard treatment does not allow full recovery from rickets or proper endochondral mineralization, providing an argument for long-term orthopedic followup of these children. This is the first identified non-ALP quantitative surrogate marker of rickets activity. MRI can be viewed as a new tool for monitoring and managing $\mathrm{XLH}$, and MRI features may be helpful in assessing the efficacy of novel rickets therapies such as anti-FGF23 antibodies [35, 40].

In children with normal ALP levels indicating metabolic control of the rickets, the abnormal physis indicates that rickets is never fully healed. This also suggests that MRI findings may reflect a longer period of time of disease activity, and that bowing may be still present despite normal ALP levels.

Bone marrow signal abnormalities, seen in 16 patients (59\%), occurred mainly in the epiphyseal part of the bone. This is a strong indicator of impaired ossification in the secondary ossification centers. Indeed, the hypothesis of mechanical stress as the cause of bone marrow edema can very likely be ruled out, since we found no correlation between the bowing of the knee and the predominant side of the signal abnormalities.

Harris lines are thought to result from episodes of relative slowing of endochondral ossification, with continued mineralization of the newly formed bone. When endochondral ossification resumes, the dense mineralized line moves away from the physis $[41,42]$. We hypothesize that the frequent occurrence of Harris lines in XLH (89\%) may be related to periods in which the treatment is less than optimal.

Some limitations and the absence of certain correlations should be highlighted. First, we found no correlation between the clinical manifestations of the disease and MRI features. While this may be due to the limited number of patients with this rare disease, it is more likely that the MRI features, similar in this regard to ALP levels, are more sensitive to variations in metabolic control than leg shape or tooth dentin mineralization. The latter require years of optimal therapy to heal $[17,43,44]$. MRI features could be a surrogate marker of recent or semirecent metabolic control of rickets in XLH, and thus of bone health. Our study aimed at the evaluation of rickets, yet it is not possible to affirm that the features we observed are XLH-specific, as we did not compare our cohort of patients with other types of rickets. Other limitations of this study were the reading of the radiographs and MRI scans by consensus, which prevented the reporting of interobserver variability, and that we did not attempt to directly compare radiographs with MRI images, since our main aim was to describe MRI findings in XLH.

Technically, MRI is not easily accessible everywhere, and acquisition takes approximately half an hour. Sedation is therefore often required in young children, and this may prevent its routine use in the follow-up of XLH in early childhood. In addition, the cost of an MRI (approximately EUR 300) is 10 times higher than that of standard radiographs of the knee (approximately EUR 30 ), which might impede its use in daily practice.

MRI allows early detection of mechanical lesions such as osteochondritis (as it did in 2 of our patients), in which the knee deformities likely play a role [45]. Our 2 patients were asymptomatic at the time of the diagnosis, however, and the clinical implications of such abnormalities have yet to be determined.

In conclusion, knee MRI provides a precise, quantitative rickets pattern that correlates well with laboratory markers of the disease. This study confirms that MRI is a valuable tool to depict rachitic changes in cartilage. In ad- 
dition, it shows that both the degree of physeal widening and its transverse extent may be useful as surrogate markers of recent or semirecent XLH activity. Until larger studies are conducted, MRI scans should be used in XLH only in the context of clinical research.

\section{Acknowledgments}

We thank the patients and their families for their contribution to the study. We also thank the Cellule de Recherche et d'Innovation of the Bicêtre Paris-Sud Hospital for supporting the preparation of the English version of the manuscript. This study was supported by the AP-HP, Bicêtre Paris-Sud, the Reference Center for Rare Disorders of Calcium and Phosphate Metabolism, and the Platform of Expertise Paris-Sud for Rare Diseases.

\section{Disclosure Statement}

A. Linglart, C. Adamsbaum, and A. Rothenbuhler report that they have received fees from Ultragenyx as investigators for a clinical trial during the period they were writing the current study. A. Rothenbuhler reports having received personal fees from Prostrakan. M. Lempicki, V. Merzoug, S. Franchi-Abella, and C. Chaussain declare no conflicts of interest.

\section{Author Contributions}

M. Lempicki, V. Merzoug, and C. Adamsbaum performed the radiographs and MRI scans; $\mathrm{M}$. Lempicki and C. Adamsbaum did the analysis of MRI and radiography images. C. Chaussain provided data on oral health. A. Rothenbuhler and A. Linglart recruited the patients and provided the clinical data. M. Lempicki, C. Adamsbaum, and A. Linglart analyzed the results and wrote the first version of the manuscript. All authors contributed to the writing and editing of the manuscript.

\section{References}

1 Carpenter TO: The expanding family of hypophosphatemic syndromes. J Bone Miner Metab 2012;30:1-9.

- Albright F, Butler AM, Bloomberg E: Rickets resistant to vitamin D therapy. Am J Dis Child 1937;54:529-547.

3 Endo I, Fukumoto S, Ozono K, Namba N, Inoue $\mathrm{D}$, Okazaki R, Yamauchi M, Sugimoto T, Minagawa M, Michigami T, Nagai M, Matsumoto T: Nationwide survey of fibroblast growth factor 23 (FGF23)-related hypophosphatemic diseases in Japan: prevalence, biochemical data and treatment. Endocr J 2015;62:811-816.

4 Gaucher C, Walrant-Debray O, Nguyen TM, Esterle L, Garabédian M, Jehan F: PHEX analysis in 118 pedigrees reveals new genetic clues in hypophosphatemic rickets. Hum Genet 2009;125:401-411.

5 A gene (PEX) with homologies to endopeptidases is mutated in patients with X-linked hypophosphatemic rickets. The HYP Consortium. Nat Genet 1995;11:130-136.

-6 Martin A, David V, Li H, Dai B, Feng JQ, Quarles LD: Overexpression of the DMP1 C-terminal fragment stimulates FGF23 and exacerbates the hypophosphatemic rickets phenotype in Hyp mice. Mol Endocrinol 2012;26:1883-1895.

7 Martin A, Liu S, David V, Li H, Karydis A, Feng JQ, Quarles LD: Bone proteins PHEX and DMP1 regulate fibroblastic growth factor Fgf23 expression in osteocytes through a common pathway involving FGF receptor (FGFR) signaling. FASEB J 2011;25:25512562.
8 Shimada T, Hasegawa H, Yamazaki Y, Muto T, Hino R, Takeuchi Y, Fujita T, Nakahara K, Fukumoto S, Yamashita T: FGF-23 is a potent regulator of vitamin D metabolism and phosphate homeostasis. J Bone Miner Res 2004;19: 429-435.

-9 Fukumoto S, Shimizu Y: Fibroblast growth factor 23 as a phosphotropic hormone and beyond. J Bone Miner Metab 2011;29:507-514.

10 Yamazaki Y, Okazaki R, Shibata M, Hasegawa Y, Satoh K, Tajima T, Takeuchi Y, Fujita T, Nakahara K, Yamashita T, Fukumoto S: Increased circulatory level of biologically active full-length FGF-23 in patients with hypophosphatemic rickets/osteomalacia. J Clin Endocrinol Metab 2002;87:4957-4960.

16 Carpenter TO, Imel EA, Holm IA, Jan de Beur SM, Insogna KL: A clinician's guide to Xlinked hypophosphatemia. J Bone Miner Res 2011;26:1381-1388.

17 Boukpessi T, Septier D, Bagga S, Garabedian M, Goldberg M, Chaussain-Miller C: Dentin alteration of deciduous teeth in human hypophosphatemic rickets. Calcif Tissue Int 2006;79:294-300.

18 Linglart A, Biosse-Duplan M, Briot K, Chaussain C, Esterle L, Guillaume-Czitrom S, Kamenicky P, Nevoux J, Prié D, Rothenbuhler A, Wicart $P$, Harvengt P: Therapeutic management of hypophosphatemic rickets from infancy to adulthood. Endocr Connect 2014; 3:R13-R30

-11 Santos F, Fuente R, Mejia N, Mantecon L, Giland growth. Pediatr Nephrol 2012;28:595603.

12 Quarles LD: FGF23, PHEX, and MEPE regulation of phosphate homeostasis and skeletal mineralization. Am J Physiol Endocrinol Metab 2003;285:E1-E9.

13 Tiosano D, Hochberg Z: Hypophosphatemia: the common denominator of all rickets. J Bone Miner Metab 2009;27:392-401.

14 Penido MG, Alon US: Phosphate homeostasis and its role in bone health. Pediatr Nephrol 2012;27:2039-2048.

15 Barros NM, Hoac B, Neves RL, Addison WN, Assis DM, Murshed M, Carmona AK, McKee MD: Proteolytic processing of osteopontin by PHEX and accumulation of osteopontin fragments in Hyp mouse bone, the murine model of X-linked hypophosphatemia. J Bone Miner Res 2013;28:688-699.
9 Fujiwara M, Namba N, Ozono K, Arisaka O, Yokoya S; Committee on Drugs, Japanese Society for Pediatric Endocrinology: Treatment of hypophosphatemic rickets with phosphate and active vitamin D in Japan: a questionnaire-based survey. Clin Pediatr Endocrinol 2013;22:9-14.

20 Harrell RM, Lyles KW, Harrelson JM, Friedman NE, Drezner MK: Healing of bone disease in X-linked hypophosphatemic rickets/ osteomalacia. Induction and maintenance with phosphorus and calcitriol. J Clin Invest 1985;75:1858-1868.

21 Mackie EJ, Tatarczuch L, Mirams M: The skeleton: a multi-functional complex organ. The growth plate chondrocyte and endochondral ossification. J Endocrinol 2011;211: 109-121.

22 Shore RM, Chesney RW: Rickets: part I. Pediatr Radiol 2012;43:140-151. 
23 Shore RM, Chesney RW: Rickets: part II. Pediatr Radiol 2012;43:152-172.

-24 Laor T, Jaramillo D: MR imaging insights into skeletal maturation: what is normal? Radiology 2009;250:28-38.

25 Keats TE, Anderson MW: Atlas of Normal Roentgen Variants That May Simulate Disease: Expert Consult. Philadelphia, Elsevier Saunders, 2012.

26 Oestreich AE: The acrophysis: a unifying concept for enchondral bone growth and its disorders. I. Normal growth. Skeletal Radiol 2003;32:121-127.

-27 Tsai A, McDonald AG, Rosenberg AE, Stamoulis C, Kleinman PK: Discordant radiologic and histological dimensions of the zone of provisional calcification in fetal piglets. Pediatr Radiol 2013;43:1606-1614.

-28 Thacher TD, Fischer PR, Pettifor JM, Lawson JO, Manaster BJ, Reading JC: Radiographic scoring method for the assessment of the severity of nutritional rickets. J Trop Pediatr 2000;46:132-139.

29 Waitches G, Zawin JK, Poznanski AK: Sequence and rate of bone marrow conversion in the femora of children as seen on MR imaging: are accepted standards accurate? AJR Am J Roentgenol 1994;162:1399-1406.

30 Adamsbaum C: Imagerie pédiatrique et foetale. Paris, Médecine Sciences Flammarion, 2007.

31 Ecklund K, Doria AS, Jaramillo D: Rickets on MR images. Pediatr Radiol 1999;29:673-675.
32 Imel EA, DiMeglio LA, Hui SL, Carpenter TO, Econs MJ: Treatment of X-linked hypophosphatemia with calcitriol and phosphate increases circulating fibroblast growth factor 23 concentrations. J Clin Endocrinol Metab 2010;95:1846-1850.

33 Petje G, Meizer R, Radler C, Aigner N, Grill F: Deformity correction in children with hereditary hypophosphatemic rickets. Clin Orthop Relat Res 2008;466:3078-3085.

34 Miyamoto J, Koto S, Hasegawa Y: Final height of Japanese patients with X-linked hypophosphatemic rickets: effect of vitamin D and phosphate therapy. Endocr J 2000;47:163167.

35 Carpenter TO, Imel EA, Ruppe MD, Weber TJ, Klausner MA, Wooddell MM, Kawakami T, Ito T, Zhang X, Humphrey J, Insogna KL, Peacock M: Randomized trial of the antiFGF23 antibody KRN23 in X-linked hypophosphatemia. J Clin Invest 2014;124:15871597.

36 Study of KRN23, a recombinant fully human monoclonal antibody against FGF23, in pediatric subjects with $\mathrm{X}$-linked hypophosphatemia (XLH) - full text view - ClinicalTrials.gov (cited June 16, 2014]. Available from: https:// clinicaltrials.gov/ct2/show/NCT02163577? term $=\mathrm{krn} 23 \& \mathrm{rank}=3$.

37 Kraus R, Reyers J, Alt V, Schnettler R, Berthold LD: Physiological closure of the physeal plate of the distal radius: an MRI analysis. Clin Anat 2011;24:1010-1015.

38 Villemure I, Stokes IAF: Growth plate mechanics and mechanobiology. A survey of present understanding. J Biomech 2009;42: 1793-1803.
39 Donohue MM, Demay MB: Rickets in VDR null mice is secondary to decreased apoptosis of hypertrophic chondrocytes. Endocrinology 2002;143:3691-3694.

-40 Aono Y, Yamazaki Y, Yasutake J, Kawata T, Hasegawa H, Urakawa I, Fujita T, Wada M, Yamashita T, Fukumoto S, Shimada T: Therapeutic effects of anti-FGF23 antibodies in hypophosphatemic rickets/osteomalacia. J Bone Miner Res 2009;24:1879-1888.

41 Jaramillo D, Shapiro F: Growth cartilage: normal appearance, variants and abnormalities. Magn Reson Imaging Clin N Am 1998;6:455471.

42 Papageorgopoulou C, Suter SK, Rühli FJ, Siegmund F: Harris lines revisited: prevalence, comorbidities, and possible etiologies. Am J Hum Biol 2011;23:381-391.

43 Chaussain-Miller C, Sinding C, Septier D, Wolikow M, Goldberg M, Garabedian M: Dentin structure in familial hypophosphatemic rickets: benefits of vitamin $\mathrm{D}$ and phosphate treatment. Oral Dis 2007;13:482-489.

44 Gaucher C, Boukpessi T, Septier D, Jehan F, Rowe PS, Garabédian M, Goldberg M, Chaussain-Miller C: Dentin noncollagenous matrix proteins in familial hypophosphatemic rickets. Cells Tissues Organs 2009;189:219-223.

45 Jacobi M, Wahl P, Bouaicha S, Jakob RP, Gautier E: Association between mechanical axis of the leg and osteochondritis dissecans of the knee: radiographic study on 103 knees. Am J Sports Med 2010;38:1425-1428. 